\section{References}

1. Dick AW, Perencevich EN, Pogorzelska-Maziarz M, et al. A decade of investment in infection prevention: a cost-effectiveness analysis. Am J Infect Control 2015;43:4-9.

2. Weber DJ, Anderson D, Rutala WA. The role of the surface environment in healthcare-associated infections. Curr Opin Infect Dis 2013;26:338-344.

3. Barker AK, Alagoz O, Safdar N. Interventions to reduce the incidence of hospital-onset Clostridium difficile infection: an agent-based modeling approach to evaluate clinical effectiveness in adult acute care hospitals. Clin Infect Dis 2018;66:1192-1203.

4. Orenstein R, Aronhalt KC, McManus JE, et al. A targeted strategy to wipe out Clostridium difficile. Infect Control Hosp Epidemiol 2011;32:1137-1139.

5. Cadnum JL, Hurless KN, Kundrapu S, Donskey CJ. Transfer of Clostridium difficile spores by non-sporicidal wipes and improperly used hypochlorite wipes: practice + product $=$ perfection. Infect Control Hosp Epidemiol 2013;34:441-442.
6. Fitzgerald T, Sholtz LA, Marion N, Turner P, Carling PC, Rupp ME. Maintenance of environmental services cleaning and disinfection in the ICU after a performance improvement project. Am J Infect Control 2012;40:e159.

7. Tyan K, Jin K, Kang J. Novel colour additive for bleach disinfectant wipes reduces corrosive damage on stainless steel. J Hosp Infect (in press).

8. Lower and upper RLU limits for ATP monitoring programs. Hygiena website. https://www.hygiena.com/hc-getstarted.html. Published 2016. Accessed November 21, 2018.

9. Mustapha A, Cadnum JL, Alhmidi H, Donskey CJ. Evaluation of novel chemical additive that colorizes chlorine-based disinfectants to improve visualization of surface coverage. Am J Infect Control 2018;46:119-121.

10. Tyan K, Kang J, Jin K, Kyle AM. Evaluation of the antimicrobial efficacy and skin safety of a novel color additive in combination with chlorine disinfectants. Am J Infect Control 2018;46:1254-1261.

\title{
A regional collaboration between competing healthcare systems to establish influenza season parameters
}

\author{
David H. Priest MD, MPH ${ }^{1}$, Susan L. DeCamp-Freeze RN, MBA ${ }^{1}$, Cynthia B. Snider MD, MPH ${ }^{2}$, Melissa G. Morgan \\ MSN, RN ${ }^{2}$, Misty D. Garner MLS ${ }^{3}$, Laurence B. Givner MD ${ }^{4}$, Catherine L. Passaretti $\mathrm{MD}^{5}$ and \\ Andrea B. McQuaigue $\mathrm{RN}^{6}$ \\ ${ }^{1}$ Department of Infection Prevention, Novant Health, Winston-Salem, North Carolina, ${ }^{2}$ Department of Infection Prevention, Cone Health, Greensboro, North \\ Carolina, ${ }^{3}$ Department of Infection Prevention, Randolph Health, Asheboro, North Carolina, ${ }^{4}$ Department of Pediatrics, Wake Forest Baptist Health, Winston- \\ Salem, North Carolina, ${ }^{5}$ Department of Infection Prevention, Atrium Health, Charlotte, North Carolina and ${ }^{6}$ Department of Infection Prevention, High Point \\ Regional UNC Health Care, High Point, North Carolina
}

To the Editor-Collaborative public health partnerships between competing healthcare system hospitals are uncommon in today's healthcare environment, despite their potential for improving community health. ${ }^{1-4}$ One potential area of collaboration between hospital systems is management of influenza season parameters and messaging team members and the community. Traditionally, acute-care facilities make independent decisions regarding influenza season parameters. In geographic areas with multiple healthcare systems, the lack of coordination in influenza-season decision making can lead to a local patchwork of policies and messages. These different public health messages can be confusing to patients and the public, potentially affecting patient care, visitor access, and patient satisfaction.

We established a regional collaboration between multiple healthcare systems that emphasized information sharing and unified messaging to the public and local media. A weekly conference call was initiated that included 6 competing healthcare entities located in the piedmont region of North Carolina: Novant Health, Cone Health, Randolph Health, Wake Forest Baptist Health, Atrium Health, and High Point Regional UNC Health Care. In total, these systems represent 36 acute-care facilities covering $\sim 9,000$ square miles with $\sim 4$ million people. ${ }^{5}$ All of these systems have mandatory influenza vaccination programs for team

Author for correspondence: David H. Priest, MD, MPH, 1381 Westgate Center Drive, Winston-Salem, NC 27103. E-mail: dhpriest@novanthealth.org

Cite this article: Priest DH, et al. (2019). A regional collaboration between competing healthcare systems to establish influenza season parameters. Infection Control \& Hospital Epidemiology 2019, 40, 258-260. doi: 10.1017/ice.2018.297 members, and 4 of the systems have mandatory masking programs for team members unable to be vaccinated (ie, Novant Health, Atrium Health, High Point Regional UNC Health Care, and Randolph Health). The call included infection prevention representatives, marketing/communication professionals, nursing leaders, physicians, and hospital administrators. Each healthcare system reported their local emergency department influenza-likeillness (ILI) rate for comparison with the other systems. Facility differences were noted based on the geographic location of the hospitals. Emergency department (ED) ILI data were obtained using the North Carolina Disease Event Tracking and Epidemiologic Collection Tool (NC DETECT), a statewide syndromic surveillance system. ${ }^{6}$ Collective decisions were made to establish parameters for influenza season, which were then communicated through internal and external communication pathways.

Influenza season was declared when the ILI rate in North Carolina hospital ED visits reached $\geq 5 \%$ and ended when the ILI rate declined back to $<5 \%$ (Fig. 1), considering the general ILI trend. For the 2017-2018 influenza season, the start date was December 27, 2017, and the end date was March 30, 2018. Declaring when influenza season started and ended was more important for those healthcare systems that require mandatory masking of team members who do not receive an influenza vaccination, as this was the date in which mandatory masking began. In the past, for some healthcare systems, the influenza season started and ended using predetermined dates such as October 1 (start) and March 31 (end). Although the time between these predetermined dates typically encompassed the influenza season, 


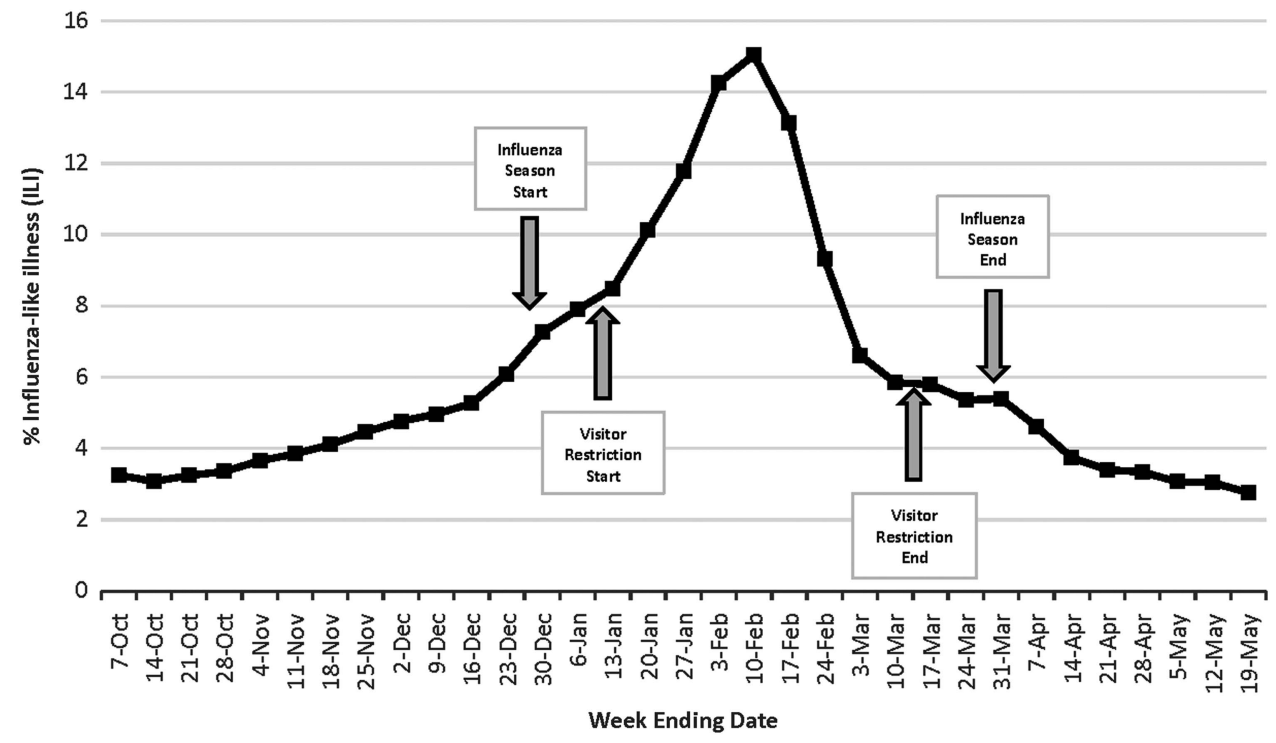

Fig. 1. Influenza-like illness surveillance, North Carolina Emergency Departments, 2017-2018. Near real-time syndromic surveillance for ILI is conducted through the North Carolina Disease Event Tracking and Epidemiologic Collection Tool (NC DETECT). This system uses a variety of data sources including emergency departments (EDs). NC DETECT is currently receiving data daily from 126 of the $12624 / 7$ emergency departments in North Carolina. The NC DETECT ILI syndrome case definition includes any case with the term "flu" or "influenza," or at least 1 fever term and 1 influenza-related symptom.

these dates did not consider variations in influenza activity in a given year. Using predetermined influenza season dates instead of a data-based approach has the potential to lead to team member masking early in the season when it might not be necessary or to the discontinuation of masking later when influenza activity remains high. Our approach allowed for masking only during times of true increased influenza activity and allowed for extension of the traditional influenza season if warranted.

Visitor restrictions, defined as the restriction of visitation to an acute-care facility by children $<13$ years of age, was instituted by all healthcare systems when the ILI rate was $\geq 7 \%$ and lifted when the ILI rate returned to $<7 \%$ (Fig. 1). For the 2017-2018 influenza season, visitor restrictions were initiated on January 12, 2018, and lifted on March 16, 2018. When the $\geq 7 \%$ threshold was reached, a coordinated effort was made between the healthcare systems to provide consistent messaging to team members and the media about who would be restricted from acute-care facilities and when. This was particularly effective for communicating with local media outlets as the media reported the collective decision using a single story. ${ }^{7,8}$ In years past, multiple media stories would have been published as each system made their decision, leading to mixed public messaging. Likewise, when the level of influenza activity fell to $<7 \%$, the media was informed that visitor restrictions would be lifted. We found that marketing and communication professionals were vital to the success of this process.

One additional benefit was noted. In our communities, team members often work in multiple facilities moving between the different healthcare systems. A unified approach to the influenza season reduced confusion when team members moved between hospitals.

Our approach has some limitations. The chosen thresholds for our decisions were based on clinical judgement and not published research. In addition, the size of some of the healthcare organizations meant that a few hospitals were geographically distant. Thus, the ILI rate near those facilities might vary significantly from the other facilities in the collaborative. This distance has led to the consideration of developing additional collaboratives in those areas.
The development of this process was a simple solution that improved communication between competing healthcare systems, simplified public messaging around influenza season, and standardized influenza season parameters in a single geographic region. The collaboration and exchange of information also increased the congeniality between the healthcare systems, and the participants found it to be informative and enjoyable. We hope that we can apply this approach to additional issues to benefit the communities that we serve.

Acknowledgments. The case data used in this study were provided by the North Carolina Disease Event Tracking and Epidemiologic Collection Tool (NC DETECT), an advanced, statewide public health surveillance system. NC DETECT is funded with federal funds by North Carolina Division of Public Health (NC DPH), Public Health Emergency Preparedness Grant (PHEP), and is managed through a collaboration between NC DPH and the University of North Carolina at Chapel Hill Department of Emergency Medicine's Carolina Center for Health Informatics (UNC CCHI).

Financial support. No financial support was provided relevant to this article.

Conflicts of interest. All authors report no conflicts of interest relevant to this article.

\section{References}

1. Prybil LD, Scutchfield FD, Dixon RE. The evolution of public health-hospital collaboration in the United States. Pub Health Rep 2016;131:522-525.

2. Roussos ST, Fawcett SB. A review of collaborative partnerships as a strategy for improving community health. Annu Rev Pub Health 2000;21:369-402.

3. Hardin L, Kilian A, Spykerman K. Competing health care systems and complex patients: an inter-professional collaboration to improve outcomes and reduce health care costs. J Interprof Educ Pract 2017;7:5-10.

4. Ainsworth D, Diaz H, Schmidtlein MC. Getting more for your money: designing community needs assessments to build collaboration and capacity in hospital system community benefit work. Health Promot Pract 2012;14:868-875.

5. United States Census Bureau website. https://www.census.gov/quickfacts/ nc. Published 2017. Accessed October 2, 2018. 
6. The North Carolina Disease Event Tracking and Epidemiologic Collection Tool (NC DETECT) website. https://ncdetect.org/. Accessed October 1, 2018.

7. Several healthcare systems restricting visitors due to flu. WCNC television station website. https:/www.wcnc.com/article/news/health/several-health care-systems-restricting-visitors-due-to-flu/275-507119056.

Published

2018. Accessed September 26, 2018.

8. Charlotte hospitals to lift flu ban on kids. Here's what it means for visitors. The Charlotte Observer website. https://www.charlotteobserver.com/news/local/ article205266564.html. Published 2018. Accessed September 26, 2018.

\title{
Impact of expanded influenza post-exposure prophylaxis on healthcare worker absenteeism at a tertiary care center during the 2017-2018 season
}

\author{
Mireia Puig-Asensio MD, PhD, Margaret Douglas RRT, MPH, Stephanie Holley MBA, BSN, Mary E. Kukla BSN, RN, \\ Oluchi Abosi MPH, MBChB, Lisa Mascardo PharmD, Brenda Carmody BS Pharm RPh, Courtney Gent PharmD, \\ Daniel J. Diekema MD, MS, Patrick Hartley MB, BCh, BAO, Michael B. Edmond MD, MPH, MPA and
}

Jorge L. Salinas MD

University of Iowa Hospitals \& Clinics, lowa City, lowa

To the Editor-Healthcare workers (HCWs) are at risk of being exposed to influenza during routine patient care. ${ }^{1}$ Consequently, HCW vaccination is advised to reduce influenza-related morbidity and absenteeism. ${ }^{2,3}$ However, influenza vaccine effectiveness varies from season to season depending on the level of vaccine matching with circulating influenza strains.

During 2017-2018, there was an expected predominance of influenza $\mathrm{H} 3 \mathrm{~N} 2$, a subtype associated with lower vaccine effectiveness. We hypothesized that a surge in influenza cases paired with decreased vaccine effectiveness could increase HCW absenteeism and impact the delivery of care. Historically, we offered oseltamivir postexposure prophylaxis (PEP) only to unvaccinated exposed HCWs, but during the 2017-2018 season, we expanded PEP to all exposed HCWs regardless of their vaccination status. We report our experience describing PEP uptake, cost, and impact on HCW absenteeism at the University of Iowa Hospitals \& Clinics (UIHC) during 2 influenza seasons (2016-2017 and 2017-2018).

The UIHC is an 811-bed tertiary-care hospital that serves as a referral and safety-net health system for eastern Iowa. During the 2016-2017 season, PEP (75 mg/day for 7 days) was offered free of charge to unvaccinated exposed HCWs. We defined exposure as proximity within 3 feet of a laboratory-confirmed influenza-infected person for $\geq 10$ minutes without mask protection, or direct contact with respiratory secretions. Prophylaxis was not recommended if $>48 \mathrm{~h}$ had elapsed since the exposure. During the 2017-2018 season, PEP was expanded to all exposed HCWs regardless of vaccination status. Other hospital infection control policies did not change over the study period: (1) Universal surgical mask use was advised for HCWs with direct patient contact in hematology-oncology units during respiratory virus season. (2) Oseltamivir prophylaxis was

Author for correspondence: Mireia Puig-Asensio, Division of Infectious Diseases, Internal Medicine Department, University of Iowa Hospitals \& Clinics, 200 Hawkins Drive, Iowa City, IA 52246. E-mail: mireia-puigasensio@uiowa.edu

Cite this article: Puig-Asensio M, et al. (2019). Impact of expanded influenza postexposure prophylaxis on healthcare worker absenteeism at a tertiary care center during the 2017-2018 season. Infection Control \& Hospital Epidemiology 2019, 40, 260-261. doi: $10.1017 /$ ice.2018.317 recommended to all patients and HCWs in units with nosocomial transmission of influenza (ie, $\geq 2$ new cases of influenza in inpatients 72 hours or more after admission). Influenza immunization status was recorded in a secure, on-line employee health portal (ReadySet, Axion Health, Westminster, CO). Influenza testing was performed by polymerase chain reaction during both seasons.

The study population was staff nursing and clinical technicians who worked at UIHC in both adult and pediatric units (inpatient and outpatient). We reviewed surveillance, employee health, pharmacy, and human resources records for the 2016-2017 and 2017-2018 seasons."Influenza season" was defined as October 1 through March 31. For HCWs with multiple exposures, only exposure events that occurred $>7$ days apart were included. The primary outcome was the rate of absenteeism, expressed as all-cause sick leave in hours divided by the total scheduled work hours. We excluded absences $<1$ hour. We defined PEP uptake as prescriptions picked up divided by the number of HCWs who were referred. Oseltamivir prophylaxis prescribed because of nosocomial transmission was not considered PEP. Analyses were conducted in Stata version 15 software (StataCorp, College Station, TX).

During the 2016-2017 and 2017-2018 seasons, we identified 373 and 427 laboratory-confirmed influenza cases, respectively. The proportion of HCWs who received the influenza vaccine was similar for both seasons (89.7\% vs $90.8 \%$ ). PEP was recommended for 15 exposed HCWs in 2016-2017 and 280 in 2017-2018, and 5 (33.3\%) and 133 (47.5\%) HCWs picked up oseltamivir from the pharmacy, respectively. Oseltamivir cost an average of $\$ 81.41$ per PEP course. The total estimated cost of oseltamivir was $\$ 407$ in 2016-2017 and \$10,828 in 2017-2018. During the 2016-2017 season, there were 6,187 sick-leave requests with a median of 12 working hours (IQR, 10-24) lost per HCW. During the 2017-2018 season, there were 6,174 sick-leave requests with a median of 12 hours (IQR, 11.6-24). Absenteeism rates were similar in both influenza seasons ( $3.2 \%$ vs $3.4 \%$, respectively).

During the 2017-2018 influenza season, our recommendation of providing PEP to all HCWs exposed to an influenza-infected case had no apparent impact on overall absenteeim rates or the 